\title{
Utility of Awake Prone Positioning with Low-Dose Systemic Corticosteroids in Coronavirus Disease 2019 Acute Respiratory Distress Syndrome Patients: A Case Series
}

\author{
Vishal Shanbhag, Souvik Chaudhuri, N. R. Arjun, Nitin Gupta ${ }^{1,2}$, Shashikiran Umakanth ${ }^{2,3}$, Kavitha Saravu ${ }^{1,2}$ \\ Departments of Critical Care Medicine and ${ }^{1}$ Infectious Diseases, Kasturba Medical College, ${ }^{2}$ Manipal Center of Infectious Diseases, Prasanna School of Public Health, \\ Manipal Academy of Higher Education, ${ }^{3}$ Department of Medicine, TMA Pai Hospital, Manipal Academy of Higher Education, Manipal, Karnataka, India
}

\section{Abstract}

Coronavirus disease 2019 (COVID-19) is caused by severe acute respiratory syndrome coronavirus 2, novel enveloped RNA beta-coronavirus. The clinical manifestations vary from no symptoms to fever, cough, respiratory distress, pneumonia, and adult respiratory distress syndrome (ARDS) or uncommonly diarrhea. Typically, the disease has viremic phase, pulmonary phase, and severe host inflammatory phase. Prompt interventions in the first two phases such as awake prone positioning and administration of low-dose corticosteroids can prevent the onset of the third phase of the deadly disease. Awake prone positioning and systemic corticosteroids are simple interventions, which can prevent the requirement of mechanical ventilation and also lead to reduction in the inflammatory response and disease severity. We report a case series of six patients who diagnosed with COVID-19 ARDS and were benefitted by awake prone positioning sessions and low-dose systemic corticosteroid therapy.

Keywords: Acute respiratory distress syndrome, awake prone positioning, coronavirus disease 2019, corticosteroids

\section{INTRODUCTION}

Management of coronavirus disease 2019 (COVID-19) patients involves timely care of two crucial aspects of the disease process, optimizing respiratory mechanics for improving hypoxia and mitigating hyperinflammatory response. Supine mechanical ventilation, with all known benefits and safety, cannot resolve the basal atelectasis, mucus plugging, and ventilation-perfusion (V/Q) mismatch. This results in a high shunt fraction, which is one of the known mechanisms of hypoxia in COVID-19 pneumonia. Awake prone positioning is a simple step which can go a long way to address these issues. ${ }^{[1-5]}$

Systemic corticosteroids, which were initially found nonbeneficial, when administered early, at low doses, reduce the hyperinflammatory markers of the disease and also have mortality benefit. ${ }^{[6,7]}$

Our case series highlights the benefits of these two simple interventions in reducing the severity of the disease process.

\begin{tabular}{|l|l|}
\hline \multicolumn{2}{|c|}{ Access this article online } \\
\hline Quick Response Code: & Website: \\
\hline & www.ijrc.in \\
\cline { 2 - 2 } & \\
\hline
\end{tabular}

\section{Case Reports}

\section{Case 1}

A 60-year-old gentleman was admitted to the hospital with complaints of cough, shortness of breath, and fever for 2 days. His nasopharyngeal swab was positive for novel coronavirus 2019 (severe acute respiratory syndrome coronavirus 2). He had a room air oxygen saturation $\left(\mathrm{SpO}_{2}\right)$ of $89 \%$ and was tachypneic with a respiratory rate (RR) of $28-30 / \mathrm{min}$. Chest $\mathrm{X}$-ray (CXR) revealed bilateral lower zone infiltrates, more on the left side, and lung ultrasonography revealed B-lines in the bilateral lower zones [Figures 1 and 2]. His inflammatory

Address for correspondence: Dr. Kavitha Saravu Department of Infectious Diseases, Kasturba Medical College, Manipal - 576 104, Karnataka, India. E-mail: kavithasaravu@gmail.com

This is an open access journal, and articles are distributed under the terms of the Creative Commons Attribution-NonCommercial-ShareAlike 4.0 License, which allows others to remix, tweak, and build upon the work non-commercially, as long as appropriate credit is given and the new creations are licensed under the identical terms.

For reprints contact: WKHLRPMedknow_reprints@wolterskluwer.com

How to cite this article: Shanbhag V, Chaudhuri S, Arjun NR, Gupta N, Umakanth S, Saravu K. Utility of awake prone positioning with low-dose systemic corticosteroids in coronavirus disease 2019 acute respiratory distress syndrome patients: A case series. Indian J Respir Care 2021;10:100-6.

Received: $13-07-2020$

Accepted: 07-09-2020 Published: $31-01-2021$ 
markers, ferritin and D-dimer, were high, being $1161 \mathrm{ng} / \mathrm{mL}$ and $2.4 \mathrm{mcg} / \mathrm{mL}$, respectively. C-reactive protein (CRP) was also raised at $253 \mathrm{mg} / \mathrm{L}$, and neutrophil-lymphocyte ratio (NLR) was $>10$. He was administered oxygen via nasal cannula (NC) at $2 \mathrm{~L} / \mathrm{min}$, and awake prone positioning was done. His $\mathrm{SpO}_{2}$ improved to $99 \%$, his tachypnea gradually settled over a period of 6 days, and inflammatory markers had a reduction from baseline levels (CRP of $124 \mathrm{mg} / \mathrm{L}$ ). He underwent $3-4$ sessions of proning per day, for about $1-2 \mathrm{~h}$ each session. He was treated with hydroxychloroquine (HCQ) and methylprednisolone (MP) $40 \mathrm{mg}$ intravenous (IV) BD for 5 days and enoxaparin $0.4 \mathrm{~mL}$ subcutaneously (SC) twice a day (BD).

He also had acute kidney injury and metabolic acidosis, hyperkalemia with creatinine of $2 \mathrm{mg} / \mathrm{dL}$, and oliguria. Since his creatinine clearance was $>30 \mathrm{~mL} / \mathrm{min}$, he was continued on enoxaparin and we did not change over to unfractionated heparin (UFH). He had symptomatic hyponatremia with altered mental status, which could be attributed to thiazide

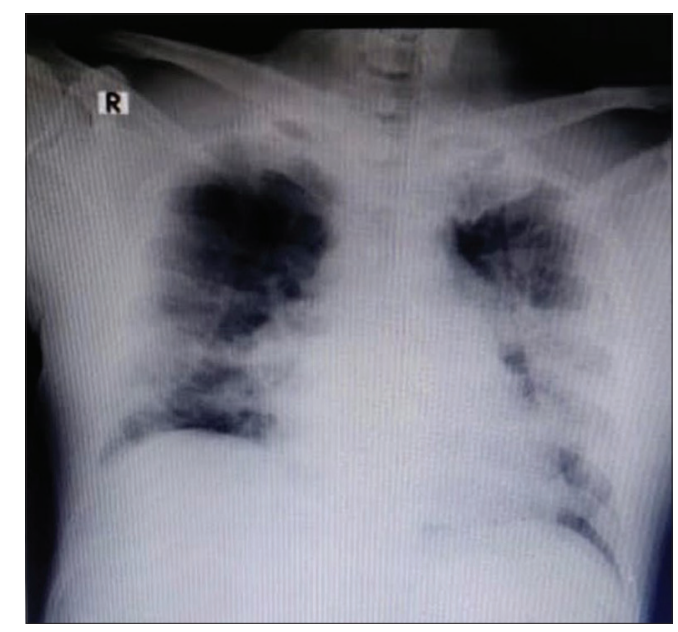

Figure 1: Chest $X$-ray showing bilateral infiltrates, predominantly in the lower zones

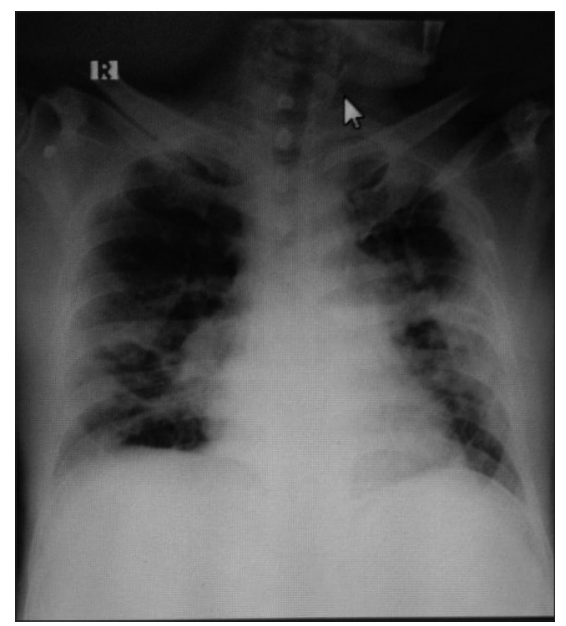

Figure 3: Chest X-ray showing resolution of the bilateral infiltrates posttreatment diuretic and ARDS. He was managed conservatively without the need for renal replacement therapy, and renal dysfunction gradually resolved with hydration as per the fluid status assessment (inferior vena cava collapsibility). HCQ was administered for 5 days and MP was also administered for 5 days. He gradually recovered from hypoxia, the CXR improved [Figure 3], and the patient was shifted out of intensive care unit (ICU).

\section{Case 2}

A 53-year-old gentleman, with a history of cough, fever, and shortness of breath and a travel history from Mumbai, was diagnosed with COVID-19 infection. His CXR showed bilateral lower zone infiltrates [Figure 4]. He had raised CRP $(253 \mathrm{mg} / \mathrm{L})$, ferritin $(1517 \mathrm{ng} / \mathrm{mL})$, D-dimer $(2.4 \mathrm{mcg} / \mathrm{mL})$, and NLR $>11$. He was tachypneic and required noninvasive ventilation (NIV). Due to delirium, he could not be persuaded for awake proning. Due to poor partial pressure of oxygen in the arterial blood $\left(\mathrm{PaO}_{2}\right)$ to fraction of inspired oxygen $\left(\mathrm{FiO}_{2}\right)$ or $(\mathrm{P} / \mathrm{F})$ ratio 180 with moderate $\mathrm{ARDS}$, he was intubated and

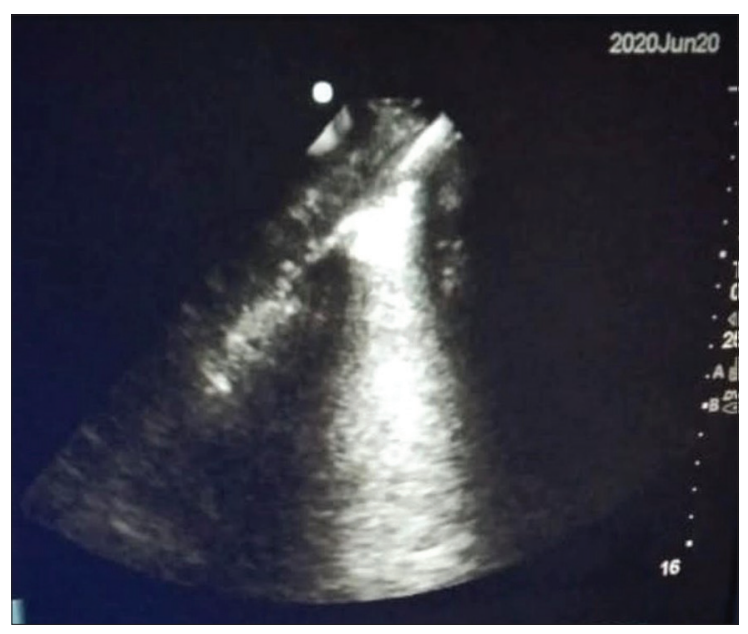

Figure 2: Lung ultrasound picture showing basal B-lines suggestive of atelectasis

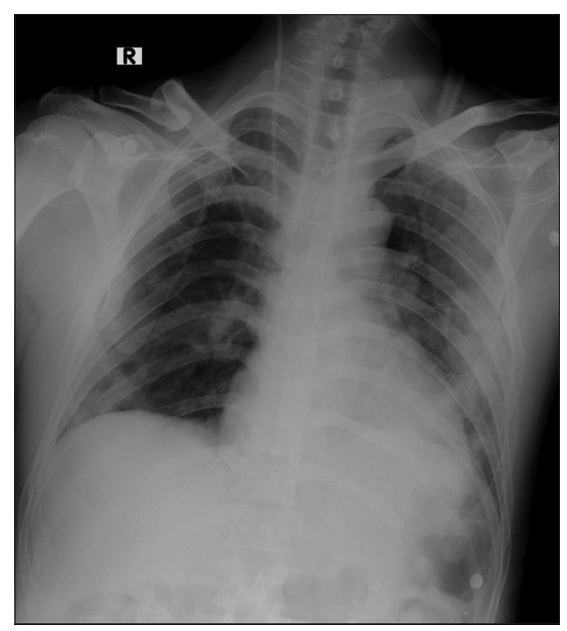

Figure 4: Chest $X$-ray depicting bilateral lower zone infiltrates, prominently on the left lower zone 
ventilated. He had good lung compliance $\left(50 \mathrm{~mL} / \mathrm{cmH}_{2} \mathrm{O}\right)$ and was probably belonging to L-phenotype. He was treated with HCQ for 5 days, MP $40 \mathrm{mg} \mathrm{BD}$ for 5 days, and enoxaparin $0.4 \mathrm{~mL}$ SC BD. Gradually, his CXR improved, his inflammatory markers reduced, and he was extubated. Postextubation, his sensorium was better, and he was maintaining $97 \% \mathrm{SpO}_{2}$ with oxygen $4 \mathrm{~L} / \mathrm{min}$. Postextubation, he was put on awake prone position about $6 \mathrm{~h}$ per day in three sessions [Figure 5]. Gradually, the oxygen requirements were tapered off, and he was shifted out of ICU. His CXR infiltrates also resolved.

\section{Case 3}

A 37-year-old gentleman, with a history of travel and contact from Mumbai, was admitted with the complaints of cough, fever, and shortness of breath. He was diagnosed to have COVID-19 by reverse transcription-polymerase chain reaction nasopharyngeal swab. His room air $\mathrm{SpO}_{2}$ was $89 \%$, and CXR revealed bilateral lung infiltrates [Figure 6]. Arterial blood gas revealed a $\mathrm{P} / \mathrm{F}$ ratio of 280 suggestive of mild ARDS. His inflammatory markers were also high, which included ferritin $(1536 \mathrm{ng} / \mathrm{mL})$, high-sensitive CRP $(6 \mathrm{mg} / \mathrm{mL})$, and lactate dehydrogenase (LDH) of $602 \mathrm{U} / \mathrm{L}$. He was administered oxygen (40\% Venturi) initially and subsequently was put on non- rebreathing face mask (NRBM) requiring almost $80 \%$ $\mathrm{FiO}_{2}$ with $\mathrm{P} / \mathrm{F}<200$. After a baseline electrocardiography to rule out contraindications, he was started on HCQ, MP $40 \mathrm{mg}$ $\mathrm{BD}$, and pantoprazole $40 \mathrm{mg}$ and ceftriaxone $2 \mathrm{~g} \mathrm{IV} \mathrm{OD} \mathrm{for}$ 5 days. He was tried on awake prone position for a total of about $14 \mathrm{~h}$ in $4-5$ sessions per day. Gradually, over a period of 5 days, his CXR showed resolution [Figure 7], his $\mathrm{P} / \mathrm{F}$ ratio improved to 450 , and inflammatory markers decreased. He was subsequently off oxygen and discharged.

\section{Case 4}

A 37-year-old woman was admitted to the hospital with fever and shortness of breath and was diagnosed as COVID-19 positive. Her CXR was unremarkable; however, she was tachypneic with a RR around $28 / \mathrm{min}$ and had a poor P/F ratio of 180 with a $\mathrm{SpO}_{2}$ of $93 \%$ on $40 \%$ Venturi. She had ferritin of $644.10 \mathrm{ng} / \mathrm{mL}, \mathrm{CRP}$ of $62 \mathrm{mg} / \mathrm{L}$, LDH of $384 \mathrm{U} / \mathrm{L}$, and D-dimer of $0.7 \mathrm{mcg} / \mathrm{mL}$. She was administered HCQ for 5 days and tablet dexamethasone $6 \mathrm{mg}$ for 5 days. As CXR was unremarkable, as per the protocol, we did not administer MP. Subsequently, with awake prone positioning sessions of about $12 \mathrm{~h}$ per day divided in four sessions, her $\mathrm{SpO}_{2}$ improved to $99 \%$ with NC of $2 \mathrm{~L} / \mathrm{min}$ and her $\mathrm{P} / \mathrm{F}$ ratio improved to 400 . With the resolution of inflammatory markers and improvement in oxygenation, she was discharged to the ward.

\section{Case 5}

A 45-year old gentleman, known hypertensive, who returned home from Mumbai, was admitted to the hospital with chief complaints of shortness of breath and fever. Nasopharyngeal swab confirmed COVID-19 positive report. He was being referred from another hospital, with 6 days of illness already. He was tachypneic with a RR of 30-35/min and CXR revealed ARDS picture with $\mathrm{P} / \mathrm{F}$ ratio revealing moderate

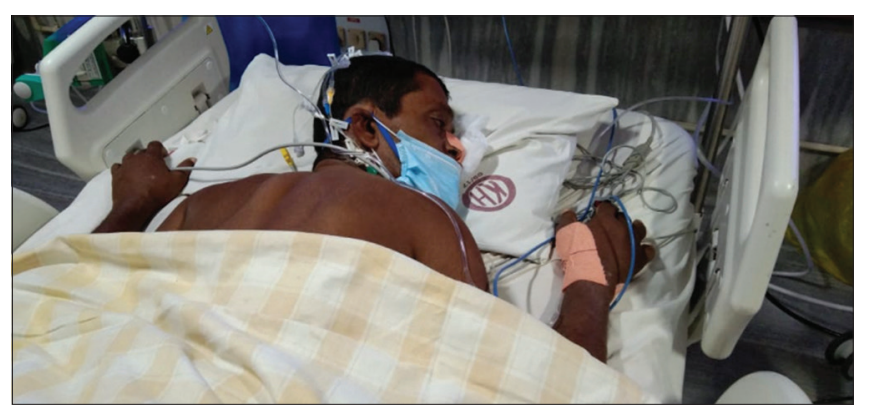

Figure 5: Awake prone positioning being done

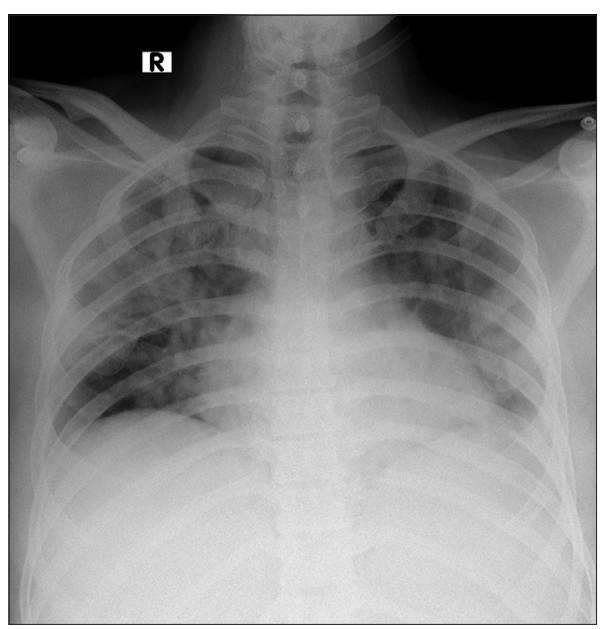

Figure 6: Chest $X$-ray showing bilateral fluffy infiltrates-adult respiratory distress syndrome picture

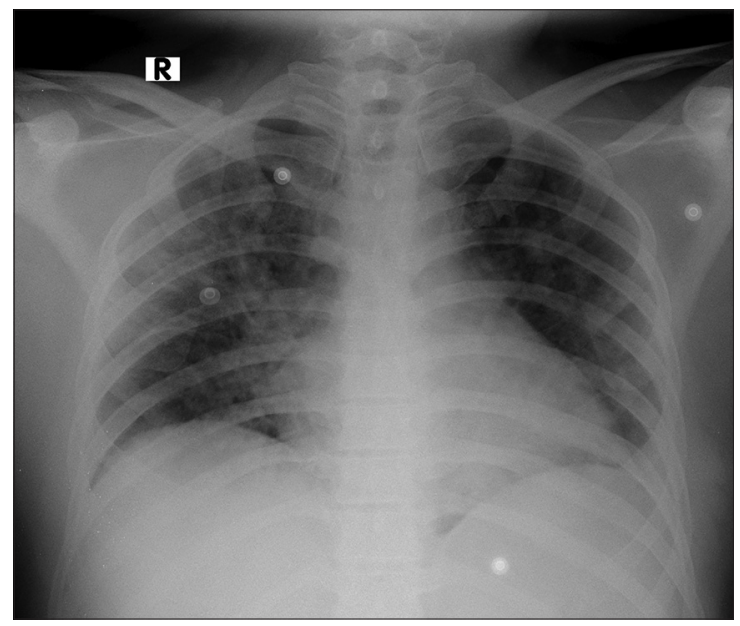

Figure 7: Resolution of the adult respiratory distress syndrome picture posttreatment

ARDS [Figure 8]. His $\mathrm{SpO}_{2}$ was $88 \%-89 \%$ on oxygen via nonrebreathing face mask (NRBM) of $15 \mathrm{~L} / \mathrm{min}$. He was intubated and ventilated in view of shock, requiring noradrenaline infusion of $6 \mathrm{~mL} / \mathrm{h}(8 \mathrm{mg}$ in $42 \mathrm{~mL}$ normal saline). On subsequent assessment, his lung compliance was low around $25 \mathrm{~mL} / \mathrm{cmH}_{2} \mathrm{O}$, classified as H-phenotype, and was ventilated with lung protective strategy, low tidal volumes, high positive end expiratory pressure (PEEP), 
and lower $\mathrm{FiO}_{2}$. His inflammatory markers were raised with CRP of $136 \mathrm{mg} / \mathrm{L}$, D-dimer of $1.3 \mathrm{mcg} / \mathrm{mL}$, LDH of 922 $\mathrm{U} / \mathrm{L}$, and ferritin of $1998 \mathrm{ng} / \mathrm{mL}$. He was started on HCQ, broad-spectrum antibiotics, and therapeutic anticoagulation as per the protocol. He was administered MP $80 \mathrm{mg}$ BD for the next 5 days. Blood cultures were sterile, and antibiotics were subsequently de-escalated. Gradually, his condition improved, CXR resolved, and he was extubated [Figure 9].

Postextubation, he was requiring NIV and $0.4-0.5 \mathrm{FiO}_{2}$, with a PEEP of $8 \mathrm{cmH}_{2} \mathrm{O}$, and he was started on awake prone sessions for about $12 \mathrm{~h}$ a day, in four sessions. During the awake proning sessions, he was either on face mask oxygen or on oxygen via NC. After 2 days, he was shifted to the medical floor where awake proning sessions were continued till he was off oxygen support. After 3 days, nasopharyngeal throat swab was negative, and he was discharged.

\section{Case 6}

A 62-year-old gentleman, a known hypertensive and chronic kidney disease (CKD) Stage V on regular hemodialysis, was diagnosed as COVID-19 positive when he presented to the

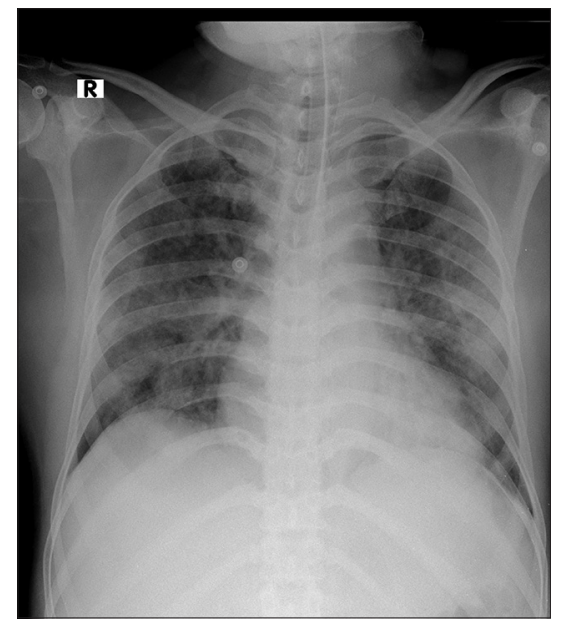

Figure 8: Bilateral nonhomogenous infiltrates involving bilateral lung zones, suggestive of adult respiratory distress syndrome

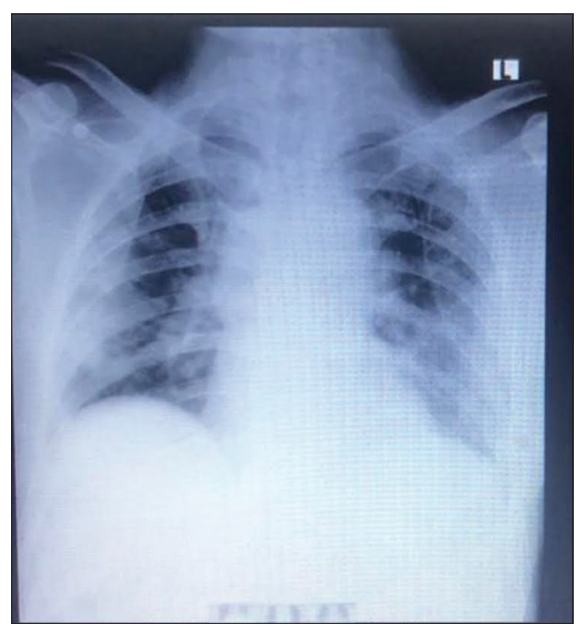

Figure 10: Bilateral lower zone infiltrates in the chest X-ray hospital with cough, high-grade fever with chills, and shortness of breath. On admission, he had a RR of 30-32/min and $\mathrm{SpO}_{2}$ of $94 \%$ on room air. Auscultation of the chest revealed bilateral crepitations. CXR showed features suggestive of bilateral peripheral infiltrates, which then progressed to ARDS [Figure 10]. He was put on $60 \%$ Venturi face mask, and $\mathrm{P} / \mathrm{F}$ ratio was 126 , suggestive of moderate ARDS. He was also oliguric, with urea of $169 \mathrm{mg} / \mathrm{dL}$ and creatinine of $10.1 \mathrm{mg} / \mathrm{dL}$. His inflammatory markers were raised, with ferritin being $>2000 \mathrm{ng} / \mathrm{mL}, \mathrm{CRP} 148 \mathrm{mg} / \mathrm{L}$, and procalcitonin $30.4 \mathrm{ng} / \mathrm{mL}$. He was administered NIV with pressure support of $5 \mathrm{cmH}_{2} \mathrm{O}$ and positive end expiratory pressure (PEEP) of $8 \mathrm{cmH}_{2} \mathrm{O}$ and underwent hemodialysis. After cultures were sent as per the sepsis protocol, he was treated with HCQ, piperacillin-tazobactam, and dexamethasone $6 \mathrm{mg}$ IV OD for 5 days and unfractionated heparin 5000 U BD. Gradually, his oxygenation levels improved, and he was administered intermittent NIV support and $40 \%$ oxygen by Venturi. He underwent awake prone positioning sessions also, about $10 \mathrm{~h}$ per day in three divided sessions. Gradually, his CXR improved [Figure 11], inflammatory markers subsided, and

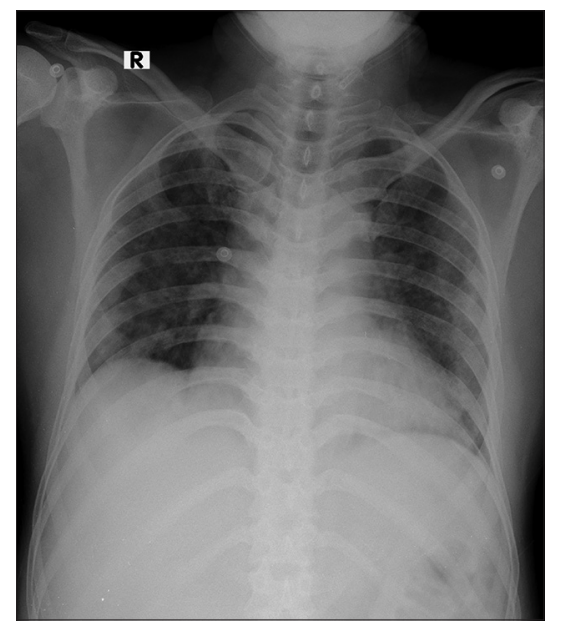

Figure 9: Resolution of the infiltrates in both lung fields posttreatment

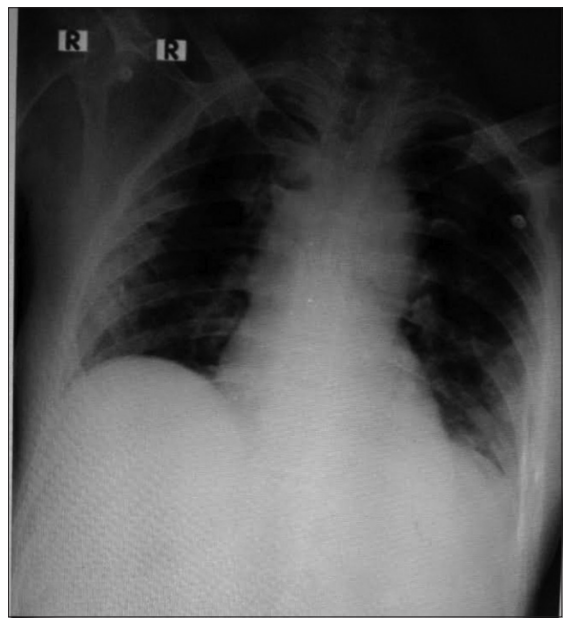

Figure 11: Chest X-ray showing dramatic improvement after treatment, with resolution of the previous lower zone infiltrates 
he was put on $\mathrm{NC}$ with oxygen $2 \mathrm{~L} / \mathrm{min}$, with $\mathrm{P} / \mathrm{F}$ ratio of 360. Culture reports were negative, and antibiotics were de-escalated after 5 days.

\section{Discussion}

COVID-19 patients often have V/Q defects as well as basal atelectasis. ${ }^{[1]}$ Autopsies have proven that the small airways are obstructed by mucus plugs. ${ }^{[1]}$ This aspect of pathophysiology of COVID-19 cannot be corrected only by optimizing ventilation in the supine position or oxygen therapy. Awake prone positioning not only is essential to improve the V/Q defects but also helps in drainage of secretions, thereby resolving the mucus plugging of airways. ${ }^{[2]}$ In the study done by $\mathrm{Xu}$ et al., awake prone positioning was attributed to be one of the most important factors, which avoided intubation in moderate ARDS patients with COVID-19. ${ }^{[2]}$ The authors further concluded that if awake prone positioning could be combined with high-flow $\mathrm{NC}$, it leads to a reduction in mortality due to respiratory failure, simultaneously being more comfortable for the patients.

Various mechanisms have been elucidated regarding the beneficial effects of prone positioning. It enhances dorsal lung aeration, relieves the compressive force on the dependent lung regions, leads to a more homogenous distribution of ventilation from ventral to dorsal lung regions, and reduces shunt fraction. ${ }^{[3,4]}$

In the case series on awake prone positioning of ten COVID-19 patients done by $\mathrm{Ng}$ et al., only one out of 10 patients required intubation. ${ }^{[4]}$ They reported that awake proning was an easy, less labor-intensive, and feasible maneuver in cooperative patients. The patients were proned for $1 \mathrm{~h}$ each session, with five proning sessions per day. A gap of $1 \mathrm{~h}$ was given postfeeds to reduce gastrointestinal side effects. This was similar to what we had requested the patients to do in our setup. They were cooperating to lie prone for about $2 \mathrm{~h}$ each session about four times a day. In a few patients, proning sessions were extended till $4 \mathrm{~h}$ and were beneficial for the patients.

In the study done by Thompson et al., on 29 hypoxemic COVID-19 patients who were administered awake prone positioning, there was improvement in oxygenation and saturation after $1 \mathrm{~h}$ of prone position, along with lesser requirement of intubation. ${ }^{[5]}$

In two of our patients in whom we had repeated lung ultrasound, there was resolution of the basal atelectasis after prone positioning as was evidenced by a significant reduction in the B-lines in the lower lung regions. The benefits of prone ventilation in severe ARDS has been validated in the proning severe ARDS (PROSEVA) trial. ${ }^{[6]}$ In the PROSEVA trial, patients with severe hypoxemia (as confirmed by a $\mathrm{P} / \mathrm{F}$ ratio of $<150 \mathrm{~mm} \mathrm{Hg}$, with an $\mathrm{FiO}_{2}$ of $\geq 0.6$ and a PEEP of $\geq 5 \mathrm{~cm} \mathrm{H}_{2} \mathrm{O}$ ), who underwent early application of prolonged prone-positioning sessions, had significantly decreased 28-day as well as 90-day mortality. ${ }^{[6]}$ The authors had concluded that prone positioning leads to a homogenous distribution of stress and strain within the lungs, decreases the overinflated lung regions, and promotes alveolar recruitment. ${ }^{[7]}$ Another aspect of treatment protocol of COVID-19 patients which has gained traction is the use of corticosteroids. The initial reports on the use of MP were less promising with a high mortality rate of about $50 \% .{ }^{[7]}$ As physicians gained more experience in the treatment of COVID-19 patients, the focus was on which steroids to administer, when, how much, and in whom? In the study by Wang et al., of 46 COVID-19 patients with pneumonia, 26 patients received low-dose MP treatment with a dosage of 1-2 $\mathrm{mg} / \mathrm{kg} /$ day for $5-7$ days via IV injection. ${ }^{[8]}$ There was rapid decrease in the inflammatory markers such as CRP and interleukin (IL)-6 in the patients after MP and was associated with better clinical outcomes in terms of oxygenation, length of ICU stay, and days of hospital stay. ${ }^{[8]}$ The most common complication of MP is onset of infection. The clinician has to be aware of the possibility of infection after treatment with systemic steroids. In case of any infection, antibiotics should be initiated without delay. ${ }^{[8]}$ The time course of illness in COVID-19 involves the viremic phase, the pulmonary phase, and the severe host inflammatory phase. MP should ideally be started early within 5 days of onset of symptoms as patients progress to the stage of pro-inflammatory storm about 5-7 days after onset. ${ }^{[8]}$ It has potent anti-inflammatory activity and has better lung tissue penetration compared to prednisolone. ${ }^{[9]}$ The cytokine surge in COVID-19 patients can be effectively attenuated by MP. ${ }^{[8]}$

The use of corticosteroid is essential and beneficial in the latter two phases of illness. Dexamethasone has shown tremendous promise in the treatment ofCOVID-19 patients in the Randomized Evaluation of COVID-19 Therapy (RECOVERY) trial. ${ }^{[10]}$ The utility of dexamethasone to mitigate immune-mediated lung injury, thereby reducing mortality rates, has been proven. ${ }^{[10]}$ Dexamethasone reduced 28-day mortality by one-third in patients receiving invasive mechanical ventilation $(29.0 \%$ vs. 40.7\%, RR 0.65 [95\% confidence interval (CI) 0.51-0.82]; $P<0.001$ ), by one-fifth in patients receiving oxygen without invasive mechanical ventilation with a dose of $6 \mathrm{mg}$ per day for up to 10 days. ${ }^{[10]}$

Before RECOVERY trial, many COVID-19 treatment guidelines stated that corticosteroids were either "contraindicated" or "not recommended" although, in China, corticosteroids are recommended for severe cases. ${ }^{[11,12]}$ However, after the RECOVERY trial, there has been a tectonic shift in the use of corticosteroids in COVID-19 patients. In all our patients, the use of awake prone positioning and corticosteroids helped in recovery from respiratory failure.

In our patients, those who had hypoxia along with infiltrates in CXR were treated with MP. MP has several advantages over hydrocortisone, in particular, in the treatment of ARDS. It has high penetration in the lung tissue with longer stay inside the tissue, has genomic and nongenomic inhibitory activity of transcription factor nuclear factor-kB, and has greater glucocorticoid receptor activity with lesser salt retaining 
(mineralocorticoid) potency. ${ }^{[13]}$ The use of MP in patients with pneumonia and ARDS in our case series proved beneficial as well. Most of our patients were weaned off ventilator or high oxygen requirement within 3-5 days after the initiation of systemic corticosteroids. The recommended dose of IV MP is $0.5-1 \mathrm{mg} / \mathrm{kg}$ and that of IV dexamethasone is $0.1-0.2 \mathrm{mg} /$ $\mathrm{kg}$, as per the guidelines issued by the Ministry of Health and Family Welfare, India. ${ }^{[14]}$

Clinicians must also be aware of the hypercoagulable state that is common in COVID-19, and hence, therapeutic anticoagulation is of utmost importance. The viral disease process leads to increase in von-Willebrand factor, Toll-like receptor activation, and tissue pathway activation. ${ }^{[15]}$ This results in raised D-dimer, fibrinogen, and cross-linked fibrin clot formation in microvasculature, which if untreated can be lethal. ${ }^{[14]}$ Heparin not only binds to COVID-19 spike protein but also reduces inflammatory mediator IL-6 level and remains the anticoagulant of choice in COVID-19 patients. ${ }^{[16]}$ For critically ill patients in ICU with COVID-19, the recommended dose of enoxaparin is $0.5 \mathrm{mg} / \mathrm{kg} \mathrm{SC}$ twice a day. ${ }^{[17]} \mathrm{In}$ a patient with creatinine clearance $<30 \mathrm{~mL} / \mathrm{min}$, the anticoagulant of choice is UFH, administered in a dose of $200 \mathrm{IU} / \mathrm{kg}$, if body mass index (BMI) $>30 \mathrm{~kg} / \mathrm{m}^{2}$ and the standard prophylactic dose for anticoagulation with UFH if BMI $<30 \mathrm{~kg} / \mathrm{m}^{2}$. ${ }^{[18]}$

This case series of six patients highlights the heterogeneity of presentation of patients of COVID-19 ARDS. It may be important, not to forget management of other comorbid illnesses, problems related to ICU admission, and severity of disease. The following aspects are of utmost significance:

1. Delirium was present in most of the patients. The contributory causes of delirium could have been prolonged ICU stay, isolation from family members, withdrawal of opioid analgesics as well as use of sedation during mechanical ventilation

It also could be a harbinger of neurotropic manifestation of severe COVID-19 disease. In our case series, every patient required some sort of pharmacological or nonpharmacological treatment of delirium, which was very important to implement successful education and self-promoted participation of patients in awake proning sessions that helped us to improve outcomes.

2. Management of the chronic health issues in COVID-19 patients should be taken care of. Many of the patients present with acute on CKD. They are predisposed to have a fluid overload state, and thus it may hasten the onset of respiratory failure in such patients. There is a challenge of balancing diuresis/fluid restrictive strategy versus risk of intravascular volume depletion precipitating vascular thrombosis, leading to increased shunt fraction. A sound knowledge of volume status assessment and risk-benefit ratio of fluid restriction in these patients is vital for improved outcomes

3. Effective communication and counseling: Effective communication with patient and family is lost somewhere either due to challenges involved in communication with personal protective equipment and/or also isolation and restriction of access to family members. We have utilized electronic media/audiovisual aids very efficiently in this regard to improve our communication with patient and family. Electronic consents, family counseling, and also psychosocial support were provided by audiovisual aids

4. Team efforts: This is the most important cornerstone for successful outcomes in COVID-19 patients with severe disease. The formation of teams and continuous discussions at different hierarchical levels is very crucial step in decision-making. A co- ordinated team effort between emergency physicians, infectious disease specialists, intensivists and respiratory therapists is of paramount importance to ensure optimal management of a patient with COVID-19.

With the management of these cases, we learned that with a holistic approach, outcomes can be really promising with timely initiation of COVID-19-specific interventions, two of which are awake proning and systemic corticosteroids as we discuss in this case series.

\section{Conclusion}

The potential utilization of early awake prone positioning with concomitant low-dose systemic corticosteroids cannot be ignored in successful management of ARDS in COVID-19 patients. Clinicians should be aware of the tremendous promise that these two simple interventions hold. These two interventions, if timely performed in cases of COVID-19 ARDS with evidence of high inflammatory response, can be cornerstones of successful outcome. Therapeutic anticoagulation should be administered in patients, especially those with a high D-dimer level to reduce the possibility of micro-thrombosis in the pulmonary vasculature.

\section{Declaration of patient consent}

The authors certify that they have obtained all appropriate consent from the patients/legally authorized representatives of the patients. The patients/legally authorized representatives have given their consent for the images and other clinical information to be reported in the journal. The patients/the legally authorized representatives understand that the patient names and initials will not be published, and due efforts will be made to conceal their identities, but anonymity cannot be guaranteed.

\section{Financial support and sponsorship}

Nil.

\section{Conflicts of interest}

There are no conflicts of interest.

\section{References}

1. Liu Q, Wang RS, Qu GQ, Wang YY, Liu P, Zhu YZ, et al. Gross examination report of a COVID-19 death autopsy. Fa Yi Xue Za Zhi 
2020;36:21-3.

2. Xu Q, Wang T, Qin X, Jie Y, Zha L, Lu W. Early awake prone position combined with high-flow nasal oxygen therapy in severe COVID-19: A case series. Crit Care 2020;24:250.

3. Albert RK, Hubmayr RD. The prone position eliminates compression of the lungs by the heart. Am J Respir Crit Care Med 2000;161:1660-5.

4. $\mathrm{Ng} \mathrm{Z}$, Tay WC, Ho CH. Awake prone positioning for non-intubated oxygen dependent COVID-19 pneumonia patients. Eur Respir J 2020;56:2001198.

5. Thompson AE, Ranard BL, Wei Y, Jelic S. Prone Positioning in Awake, Nonintubated Patients With COVID-19 Hypoxemic Respiratory Failure. JAMA Intern Med. Published online June 17, 2020. doi:10.1001/ jamainternmed.2020.3030.

6. Guérin C, Reignier J, Richard JC, Beuret P, Gacouin A, Boulain T, et al. Prone positioning in severe acute respiratory distress syndrome. N Engl J Med 2013;368:2159-68.

7. Wu C, Chen X, Cai Y, Xia J, Zhou X, Xu S, et al. Risk factors associated with acute respiratory distress syndrome and death in patients with coronavirus disease 2019 pneumonia in Wuhan, China. JAMA Intern Med 2020;180:1-11.

8. Wang Y, Jiang W, He Q, Wang C, Wang B, Zhou P, et al. A retrospective cohort study of methylprednisolone therapy in severe patients with COVID-19 pneumonia. Signal Transduct Target Ther 2020;5:57.

9. Greos LS, Vichyanond P, Bloedow DC, Irvin CG, Larsen GL, Szefler SJ, et al. Methylprednisolone achieves greater concentrations in the lung than prednisolone. A pharmacokinetic analysis. Am Rev Respir Dis 1991;144:586-92.

10. Horby P, Lim WS, Emberson J, Mafham M, Bell J, Linsell L, et al. Effect of dexamethasone in hospitalized patients with COVID-19: Preliminary report. medRxiv; 2020. Available from: https://www.medrxiv.org/content/ early/2020/06/22/2020.06.22.20137273. [Last accessed on 2020 Aug 14].

11. Dagens A, Sigfrid L, Cai E, Lipworth S, Cheng V, Harris E, et al. Scope, quality, and inclusivity of clinical guidelines produced early in the COVID-19 pandemic: Rapid review. BMJ 2020;369:m1936.

12. Zhao JP, Hu Y, Du RH, Chen ZS, Jin Y, Zhou M, et al. Expert consensus on the use of corticosteroid in patients with 2019-nCoV pneumonia. Zhonghua Jie He He Hu Xi Za Zhi 2020;43:183-4.

13. Meduri GU, Siemieniuk RA, Ness RA, Seyler SJ. Prolonged low-dose methylprednisolone treatment is highly effective in reducing duration of mechanical ventilation and mortality in patients with ARDS. J Intensive Care 2018;6:53.

14. Ministry of Health and Family Welfare. Clinical Management Protocol: COVID 19; 2020. Available from: https://www.mohfw.gov.in/pdf/ UpdatedClinicalManagementProtocolforCOVID19dated03072020. pdf. [Last accessed on 2020 Aug 14].

15. Giannis D, Ziogas IA, Gianni P. Coagulation disorders in coronavirus infected patients: COVID-19, SARS-CoV-1, MERS-CoV and lessons from the past. J Clin Virol 2020;127:104362.

16. Atallah B, Mallah SI, AlMahmeed W. Anticoagulation in COVID-19. Eur Heart J Cardiovasc Pharmacother 2020;6:260-1.

17. Barnes GD, Burnett A, Allen A, Blumenstein M, Clark NP, Cuker A, et al. Thromboembolism and anticoagulant therapy during the COVID-19 pandemic: interim clinical guidance from the anticoagulation forum. J Thromb Thrombolysis 2020;50:72-81.

18. Susen S, Tacquard CA, Godon A, Mansour A, Garrigue D, Nguyen P, et al. Prevention of thrombotic risk in hospitalized patients with COVID-19 and hemostasis monitoring. Crit Care 2020;24:364. 